\title{
Application of camelid heavy-chain variable domains (VHHs) in prevention and treatment of bacterial and viral infections.
}

WILKEN, L., MCPHERSON, A. 


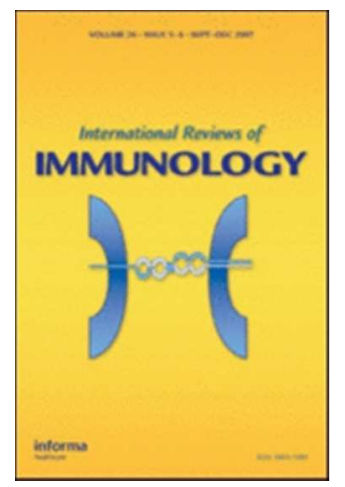

\section{Application of camelid heavy-chain variable domains (VHHs) in prevention and treatment of bacterial and viral infections}

\begin{tabular}{|r|l|}
\hline Journal: & International Reviews of Immunology \\
\hline Manuscript ID & GIRI-2017-0034.R1 \\
\hline Manuscript Type: & Review \\
\hline Complete List of Authors: & $\begin{array}{l}\text { Wilken, Lucas; Robert Gordon University, School of Pharmacy and Life } \\
\text { Sciences; Hochschule Bonn-Rhein-Sieg - Campus Rheinbach, Department } \\
\text { of Natural Sciences } \\
\text { McPherson, Anne; Robert Gordon University, School of Pharmacy and Life } \\
\text { Sciences }\end{array}$ \\
\hline Keywords: & nanobodies, immunotherapy, single-domain antibody, infectious disease \\
\hline
\end{tabular}

SCHOLARONE ${ }^{\text {M }}$

Manuscripts 
1 Lucas Wilken $^{1,2, *}$, Anne McPherson ${ }^{1}$

\section{2}

4

$5 \quad{ }^{1}$ School of Pharmacy and Life Sciences, Robert Gordon University, Garthdee

\section{Application of camelid heavy-chain variable domains (VHHs) in} prevention and treatment of bacterial and viral infections Road, Aberdeen, AB10 7GJ, United Kingdom; ${ }^{2}$ Department of Natural

Sciences, Hochschule Bonn-Rhein-Sieg, Von-Liebig-Straße 20, 53359

Rheinbach, Germany.

*Corresponding author: Lucas Wilken. E-mail: l.wilken@rgu.ac.uk. 

53

\section{Abstract}

Camelid heavy-chain variable domains (VHHs) are the smallest, intact, antigenbinding units to occur in nature. VHHs possess high degrees of solubility and robustness enabling generation of multivalent constructs with increased avidity characteristics that mark their superiority to other antibody fragments and monoclonal antibodies. Capable of effectively binding to molecular targets inaccessible to classical immunotherapeutic agents and easily produced in microbial culture, VHHs are considered promising tools for pharmaceutical biotechnology. With the aim to demonstrate the perspective and potential of VHHs for the development of prophylactic and therapeutic drugs to target diseases caused by bacterial and viral infections, this review article will initially describe the structural features that underlie the unique properties of VHHs and explain the methods currently used for the selection and recombinant production of pathogen-specific VHHs, and then thoroughly summarise the experimental findings of five distinct studies that employed VHHs as inhibitors of host-pathogen interactions or neutralisers of infectious agents. Past and recent studies suggest the potential of camelid heavy-chain variable domains as a novel modality of immunotherapeutic drugs and a promising alternative to monoclonal antibodies. VHHs demonstrate the ability to interfere with bacterial pathogenesis by preventing adhesion to host tissue and sequestering disease-causing bacterial toxins. To protect from viral infections, VHHs may be employed as inhibitors of viral entry by binding to viral coat proteins or blocking interactions with cell-surface receptors. The implementation of VHHs as immunotherapeutic agents for infectious diseases is of considerable potential and set to contribute to public health in the near future.

Key words: nanobodies, immunotherapy, single-domain antibody, infectious disease

\section{Introduction}




\section{Structure and properties of VHHs}

The production and secretion of pathogen-specific antibodies by plasma B lymphocytes

forms an integral part of the adaptive immune response to microbial infections in vertebrates. Immunoglobulin G (IgG) is the most abundant antibody type in serum and constitutes for about $75 \%$ of circulating antibodies. Due to its great availability, IgG is used as the principal antibody in immunological research. It is composed of two identical light chains and two identical heavy chains connected by disulphide bridges $[1,2]$.

Apart from conventional IgG glycoproteins with normal antibody assembly, sera of Camelidae were found to contain a high abundance of IgG subclasses, i.e. IgG $_{2}$ and $\mathrm{IgG}_{3}$, devoid of light chains. Moreover, these heavy-chain-only antibodies lack the constant heavy chain 1 domain $\left(\mathrm{C}_{H} 1\right)$ which causes direct connection of their heavy chain variable domain $\left(\mathrm{V}_{\mathrm{H}}\right)$ to the hinge. The camelid heavy-chain variable domain exhibits general structural features of a conventional $V_{H}$, but is unique in its amino acid sequence, and therefore denoted as $\mathrm{VHH}[3]$.

Equivalent to conventional $\mathrm{V}_{\mathrm{H}} \mathrm{S}, \mathrm{VHHs}$ consist of four framework regions (FRs) separating the three complementarity determining regions (CDRs) (or hypervariable regions) that are involved in antigen binding. VHHs are fully functional for antigen binding and their binding affinities are not affected by the absence of light chains. Moreover, the repertoire of antigen-binding sites is increased due to broadly sizedistributed CDRs with diverse amino acid patterns. Particularly, CDR3 of VHHs distinguishes itself from CDR3 of conventional $V_{H}$ by higher variability of its amino acid residues. CDR3 is on average longer and parts of this region that, in conventional IgG, associate with the light chain variable domain $\left(V_{L}\right)$ are available for antigen binding in VHHs. These features improve antigen recognition and binding strength, and thus compensate for the absence of the $V_{\mathrm{L}}$, which normally accounts for half of the antigen-binding surface. Furthermore, the presence of Cys residues in CDR1 and CDR3 enables the formation of disulphide bridges that stabilise the structure of the antigen-binding site [4-6]. 
VHH domains are the smallest, naturally occurring, antigen-binding units with a molecular weight of $15 \mathrm{kDa}$ [7]. Their small size allows for rapid penetration of tissues and enables construction of engineered multivalent formats with higher avidity than monoclonal antibodies (mAbs) and other antigen-binding units [8]. Moreover, solubility is notably increased in VHHs due to the presence of hydrophilic amino acid residues in FR2. Hence, these antibody fragments are resistant to aggregation and their monomeric nature is preserved in solution [9].

Amino acid substitutions cause considerable reshaping of the $\mathrm{VHH}$ surface [10] resulting in a large structural diversity of VHH paratopes, e.g. cavity, protruding loop and flat surface. Thereby, the binding of epitopes inaccessible to mAbs, such as those located in the active site of enzymes, is enabled [11].

Their small size, variability, stability, avidity and solubility characteristics render VHHs promising tools for immunotherapy.

\section{Selection and production of VHHs}

In order to obtain pathogen-specific VHHs for drug development, their generation must be artificially induced in an experimental host. For this purpose, camelids are immunised with a particular antigen isolated from the infectious organism. Peripheral blood (PB) is collected from which PB lymphocytes are prepared by density gradient centrifugation. The cells are lysed and mRNA is isolated. Extracts of mRNA are used as templates for CDNA synthesis by reverse transcription polymerase chain reaction (RTPCR). In a "nested" approach, Ig heavy chains are amplified with gene-specific primers. Reamplification of $\mathrm{VHH}$ genes with specialised primer sets that introduce restriction endonuclease (RE) recognition sites at the $5^{\prime}$ and $3^{\prime}$ ends of the RT-PCR products enables precise RE digestion of $V H H$ cDNAs [12] which are then processed for screening by phage, ribosome or yeast display [13]. For phage display, the most common of display technologies, digested products are cloned into the bacteriophage coat protein gene present in phagemid vectors [14]. The resulting recombinant DNA molecules are transformed into competent bacterial cells for gene expression. 
114 Bacterial cells are cultured to high density before infected with a helper phage [12], which provides packaging functions for phagemids, and thus, promotes phage particle formation plus their secretion into culture medium [15]. Thereby, a library of phagemid virions, each expressing a different $\mathrm{VHH}$ as fusion with their coat protein, is constructed. In a selection process, i.e. panning, VHH-displaying phage clones are applied to microtitre wells coated with the specific antigen. Adsorption of phage particles on the immobilised antigen, due to antigen-antibody interaction, is revealed by an indirect enzyme-linked immunosorbent assay (ELISA) using horseradish peroxidase-conjugated secondary antibodies directed against the phage coat protein. Vector DNA from positively selected clones is then introduced into Escherichia coli for bacterial production, with yields between 3 and $6 \mathrm{mg} \mathrm{L}^{-1}$ [12].

For increased cost efficiency, $\mathrm{VHH}$ fragments may be alternatively generated in lower eukaryotic organisms, esp. yeasts, as these are less fastidious and more productive, with secretion levels above $250 \mathrm{mg} \mathrm{L}^{-1}$. For this purpose, CDNA encoding the selected VHH is ligated into an episomal yeast expression vector. Cellular entities of Saccharomyces cerevisiae or Pichia pastoris are transformed with the recombinant vector and the pathogen-specific VHH is produced by fermentation $[16,17]$.

Subsequently, purified expressed products are subjected to antibody specificity and affinity testing by ELISA methods [12], in order to assess their suitability for further in vitro or in vivo studies.

\section{VHHs directed against bacterial pathogens}

\section{Treatment of dental caries}

Insufficient oral care, in combination with a high-sugar diet, augments the development of diverse biofilm communities on tooth surfaces, i.e. the formation of dental plaque. Acidic conditions and adhesion sites, provided by the dental plaque, favour the growth of the oral pathogen Streptococcus mutans on tooth surfaces. S. mutans further decreases $\mathrm{pH}$ by the production of acidic compounds from fermentable 
142 carbohydrates. Thereby, severe damage is caused to tooth enamel resulting in tooth 143 decay, which is symptomatic of dental caries [18-20].

144 Adherence of $S$. mutans to the salivary pellicle is mediated by the cell surface 145 streptococcal antigen (SA) I/II [21]. Prevention of adhesin-receptor interactions could 146 therefore assist in the removal of S. mutans from tooth surfaces. In 2006, Krüger et 147 al. developed an immunotherapeutic approach for the treatment of caries in patients 148 with hyposalivation that uses llama VHHs targeted against SA I/II. Krüger et al. (2006) constructed a VHH antibody library from PB lymphocytes of llamas immunised with disrupted and intact S. mutans HG982. Screening for llama VHH directed against S. mutans yielded an antibody specific to SA I/II, denoted S36VHH [20]. Previous studies had shown that coupling of VHH to enzymes enhances their therapeutic effect [22]. Therefore, by linkage of the selected VHH and Aspergillus niger-derived glucose oxidase (GOx), a fusion protein (S36-VHH-GOx) was formed to enhance the salivary peroxidase system (SPS) [20]. When glucose and oxygen are available, GOx produces hydrogen peroxide $\left(\mathrm{H}_{2} \mathrm{O}_{2}\right)$, which is required by peroxidase to oxidise the salivary components iodide $\left(\mathrm{I}^{-}\right)$and thiocyanate $\left(\mathrm{SCN}^{-}\right)$to the antibacterial compounds hypoiodite $\left(\mathrm{OI}^{-}\right)$and hypothyocyanite $\left(\mathrm{OSCN}^{-}\right)$[23]. Genes encoding S36VHH and S36-VHH-GOx were ligated into S. cerevisiae expression vectors and produced as described previously [16]. ELISAs demonstrated that the fusion of GOx to S36-VHH had no negative effect on the binding of SA I/II [20].

Therapeutic effects of these VHHs on cariogenesis were further assessed in vivo. For this purpose, the clinical situation in patients with hyposalivation was simulated by a desalivated rat model. In order to establish a basis for cariogenesis, rats received a diet high in sucrose and were repeatedly infected with S. mutans NG8. After three weeks, rats were administered with a single dose of either VHH per day. Both, S36-VHH and S36-VHH-GOx, were found to decrease colonisation with S. mutans and to exert an anticariogenic effect, however, not to statistically significant degrees. Furthermore, no additional therapeutic effect of GOx in the S36-VHH-GOx fusion protein was observed. Its nonappearance was believed to result from heavy 
plaque accumulation, partial inhibition of enzyme activity by the prevailing low $\mathrm{pH}$ as well as the lack of the electron acceptor oxygen within the plaque [20].

Although postulated, the SPS could not be enhanced. Due to virtual absence of saliva, the GOx substrate glucose was scarce, which resulted in minimal $\mathrm{H}_{2} \mathrm{O}_{2}$ generation. Consequently, the amount of oxidisable substrate, i.e. $\mathrm{I}^{-}$or $\mathrm{SCN}^{-}$, was limited leading to considerably low antibacterial effects. Krüger et al. (2006) suggest administering a mixture of S36-VHH-GOx, lactoperoxidase, and $\mathrm{I}^{-}$or $\mathrm{SCN}^{-}$, in case of hyposalivation, to achieve a bactericidal effect of the SPS.

\section{Reduction of lipopolysaccharide toxicity}

Lipopolysaccharide (LPS), or endotoxin, is a constituent of the outer membranes of gram-negative bacteria. When released into the blood stream after infection, it causes an acute inflammatory response with detrimental effects to organs and tissues, a condition described as sepsis. Sepsis results from the interaction of LPS with highaffinity LPS receptor complexes present on cells of the immune system and in plasma, which induces the release of interleukin 8 (IL-8) and other inflammatory cytokines [24-26].

An important cause of sepsis (and meningitis) is the gram-negative pathogen Neisseria meningitidis [27]. Meningococcal LPS is classified into twelve distinct immunotypes ( $L 1$ to L12) based on structural diversity of the outer core - a result of phase variation $[28,29]$. Albeit the heterogeneous structures of meningococcal LPS, its lipid A moiety and inner-core regions exhibit considerable conservation among gramnegative bacteria [30].

Protection against a variety of gram-negative bacteria could therefore be provided by immunotherapy targeting these conserved LPS components [31]. Prerequisites for the efficient use of antibodies in the prevention of sepsis comprise the recognition of an epitope located in the conserved inner core of the LPS molecule and the ability to compete with cell surface LPS receptor complexes. El Khattabi et al. explored the potential of anti-LPS VHHs for sepsis therapy in 2006. 
From PB lymphocytes of llamas, El Khattabi et al. (2006) generated a nonimmune, i.e. without immunisation of the animal, VHH phage display library. Four VHH-displaying phages that specifically bound to LPS immunotype L3 from the $N$. meningitidis strain H44/76 were selected, and purified anti-LPS VHHs were subsequently produced. All VHHs were found to immunoprecipitate meningococcal LPS forms, while some VHHs additionally reacted with LPS of other gram-negative pathogens (E. coli B4:0111, Salmonella enterica serovar Typhimurium and Bordetella pertussis). Epitope mapping was performed to determine the binding sites of the antiLPS VHHs. It was revealed that one anti-LPS VHH (VHH 5G) bound within the lipid A and KDO regions of an $r f a C$ mutant strain, while the other three reacted with an LPSdeficient mutant strain $(I p x A)$ for unknown reasons.

The effect of anti-LPS VHH on the binding of meningococcal L3 LPS by LPS receptors on innate immune cells was investigated and it was demonstrated that L3 LPS-receptor interactions could be efficiently blocked in the presence of VHH 5 G. Furthermore, it was studied whether effector cell response to LPS could be prevented by VHH 5G. LPS was effectively sequestered by VHH 5 G inhibiting the release of proinflammatory molecules. In addition, LPS depletion due to immunoprecipitation by anti-LPS VHHs was assessed to be sufficient for the detoxification of solutions contaminated with LPS [32].

With VHH 5G, an antibody was isolated that recognises LPS from different gram-negative bacteria when present in their outer membranes and in a purified, free form. VHH 5 G is able to disturb interactions between LPS and its receptors, disrupt corresponding signalling pathways that normally generate sepsis-related effector molecules, and deplete LPS from aqueous solutions with high efficiency.

\section{Prevention of enterotoxigenic E. coli-induced post-weaning diarrhoea}


Enterotoxigenic E. coli (ETEC) strains are causative of human and porcine morbidity and mortality [33] as these bacteria express heat-labile and heat-stable enterotoxins that cause ionic imbalance and secretory type diarrhoea in infected subjects $[34,35]$. ETEC virulence is determined, inter alia, by F4 fimbriae, which are filamentous protein appendages that interact with F4-specific receptors (F4Rs) present on the epithelium of the small intestine, and thereby mediate the intestinal colonisation by ETEC [36]. Intestinal isolates of ETEC from piglets with post-weaning diarrhoea (PWD) - a cause of absent maternal immunity [37] - exhibit prevalence of the serological variant F4ac [38]. In 2005, Harmsen et al. aimed to use monoclonal VHHs, raised against ETEC F4ac fimbriae, for PWD immunotherapy.

Harmsen et al. (2005) isolated F4ac fimbriae from the F4 positive (F4+) ETEC strain CVI-1000, which is devoid of F5, F6, F17, F18 and F41 fimbriae, and used these to immunise a llama and recover its VHH repertoire from PB lymphocytes. Yeast VHH expression libraries were created whence six clones, directed against the F4 fimbriae major subunit, were selected. Two clones did not significantly inhibit bacterial attachment to jejunal brush borders and displayed cross-reactivity with other F4 variants, whereas four VHHs that specifically recognised the F4ac fimbrial variant prevented F4+ ETEC attachment. The llama VHH K609 showed the strongest inhibitory activity and was therefore subjected to further studies.

First, small intestinal segments of F4R positive (F4R+) piglets, i.e. piglets with brush borders that bound above six ETEC per unit, were perfused with solutions of ETEC and different concentrations of K609. Net fluid absorption was measured to determine the effect of VHHs on ETEC-induced fluid loss. Perfusion with K609 at $4 \mathrm{mg}$ $\mathrm{L}^{-1}$ accounted for maximal reduction of ETEC-induced fluid loss, however, only to about $30 \%$. Second, faecal dry matter content was analysed in two groups of weaned piglets with severe diarrhoea, evoked by the oral challenge with porcine rotavirus strain RV277 and the ETEC strain CVI-1000. Subjects of the experimental group were orally administered daily with either low or high doses of K609, while the control group received no treatment. Faecal dry matter content of the group treated with 
257 K609 was higher compared to control piglets, but statistical significance of this

difference was merely recorded for piglets administered with high doses. Overall, reduction of diarrhoea was poor and improvement of piglet mortality was insignificant. Possible errors that may have contributed to the limited effectivity of K609 immunotherapy have been suggested. These include degradation of K609 by proteases of the gastrointestinal tract and the expression of adhesion factors, other than F4 fimbriae, by the ETEC strain CVI-1000. Furthermore, intestinal colonisation of ETEC expressing other fimbrial types or F4 variants cannot be prevented by K609 alone, owing to its specificity. It is rather suggested to produce a mixture of VHHs directed against various ETEC adhesion factors, in order to effectively obviate PWD [39].

\section{VHHs directed against viruses}

\section{Treatment of human respiratory syncytial virus infection}

Human respiratory syncytial virus (RSV) is the main contributor to lower respiratory tract infection (LRTI) in infants [40]. Patients who encountered RSV-induced bronchiolitis or pneumonia during infancy are at increased risk of developing asthma and chronic obstructive pulmonary disease in adulthood $[41,42]$. RSV specifically infects the apical membrane of ciliated respiratory epithelial cells [43] and triggers clinical symptoms of LRTI after a short incubation period [44].

RSV pathogenesis is promoted by virulence factors, esp. the envelope glycoproteins $G$ and F, encoded by a linear single-stranded, nonsegmented, negativesense RNA molecule [45]. The G protein mediates viral attachment to epithelial cell receptors [46], whereas the $\mathrm{F}$ protein induces the fusion of viral and epithelial cell membrane which enables the entry of the RSV ribonucleoprotein into host cell cytoplasm [47]. Furthermore, the F protein evokes the fusion of infected cells with adjacent cells to form multinucleated cells (syncytia) [48].

RSV is classified into two subgroups, i.e. subgroup A (RSV-A) and subgroup B (RSV-B), based on antigenic variation of the G protein [49]. In contrast, high 
conservation has been identified in the amino acid sequence of the $\mathrm{F}$ protein [50]. The development of therapeutic agents specifically targeting the F protein could therefore be highly valuable in the inhibition of viral entry. A trivalent VHH, specific to the RSV $F$ protein, was recently designed and characterised by Detalle et al. (2016) and subjected to a functional comparison with its monovalent form and the prophylactic anti-RSV mAb palivizumab [51].

Llama immune libraries were generated by injection with soluble recombinant $F$ protein, inactivated RSV-A or a combination of the two. A monovalent VHH specific to the RSV F protein (Nb017) was identified from the library. Three units of Nb017 were additionally formatted into a trivalent $\mathrm{VHH}$, denoted ALX-0171, using flexible GS linkers. Both RSV-neutralising VHHs were readily produced in a $P$. pastoris strain. Assessment of the binding to the RSV F protein, by surface plasmon resonance analysis, revealed that both ALX-0171 and Nb017 bind its pre-fusion conformation, where the trimeric format exhibited a marked increase in binding affinity. Furthermore, the effect of trimeric formatting on RSV neutralisation capacity was determined by microneutralisation assays. The potency of the trivalent ALX-0171 against RSV-A and RSV-B strains was found to be several thousandfold higher than that of the monovalent Nb017. Moreover, a significant increase in potency, as compared to palivizumab, was ascertained.

The capabilities of ALX-0171 and palivizumab to completely suppress RSV replication were compared at equivalent concentrations. ALX-0171 caused complete blockage of virus replication in $87 \%$ of viruses tested, whereas palivizumab reduced viral titres by only $18 \%$. By studying the binding to RSV mutants with alterations in antigenic site II or IV of the RSV F protein, it was demonstrated that ALX-0171 specifically targets antigenic site II. Furthermore, it was observed that ALX-0171 partially competes with palivizumab for the binding of the RSV F protein which suggests overlap of their epitopes.

As Detalle et al. (2016) intended to administer ALX-0171 by nebulisation, it was determined whether the nebulisation process causes aggregation, fragmentation 
or reduced potency of ALX-0171. Higher- and lower-molecular-weight species were detected at minimum levels, but ALX-0171 potency remained unaffected.

The in vivo efficacy of ALX-0171 against RSV was studied in cotton rats. ALX0171 was administered at different doses either by nebulisation before or intranasally after RSV challenge. Viral loads in the nose and lungs were significantly reduced for all doses of intranasally administered ALX-0171. Delivered prophylactically via nebulisation, ALX-0171 reduced nasal RSV titres in a dose-dependent matter and in the lungs it completely blocked RSV replication, even at the lowest dose tested (1 $\mathrm{mg}$ $\left.\mathrm{kg}^{-1}\right) . A L X-0171$ is therefore superior to palivizumab, which had no effect on viral titres at doses lower than $15 \mathrm{mg} \mathrm{kg}^{-1}$.

ALX-0171 is a potential therapeutic VHH that specifically and efficiently binds antigenic site II of the RSV F protein. In comparison to Nb017 and palivizumab, ALX0171 distinguishes itself by increased neutralisation capacity and inhibition efficiency, which are a result of its trivalency. Moreover, administration by nebulisation enables direct delivery of ALX-0171 to the site of infection and hence a faster exertion of its antiviral effect [52].

In May 2016, positive results for ALX-0171 in Phase I/IIa clinical trials were reported by the Belgian biopharmaceutical company Ablynx [53].

\section{Eradication of poliovirus-induced infantile paralysis (poliomyelitis)}

Poliomyelitis, or infantile paralysis, is a highly contagious disease caused by infection with poliovirus (PV) [54]. Three distinct serotypes, PV1, PV2 and PV3, all of which cause paralytic disease, have been identified based on differences in their antigenic determinants [55]. The single-stranded positive sense RNA genome of PV [56] is enclosed in a non-enveloped capsid composed of 60 monomers of four different polypeptides, i.e. viral protein 1 (VP1), VP2, VP3 and VP4 [57], that are arranged in icosahedral symmetry [58]. PV recognises and binds CD155, a transmembrane glycoprotein of the Ig superfamily [59]. Extracellular domains of CD155 interact with a conserved narrow surface depression in the PV capsid, termed canyon [60], inducing a 
conformational change of the virion that initiates cell entry and uncoating $[61,62]$. In

rare cases, PV reaches the central nervous system where it replicates in motor neurons of the spinal cord and thereby causes muscle paralysis [63].

Prophylactic treatment by inactivated polio vaccine (IPV) [64] and oral polio vaccine [65] led to near eradication of poliomyelitis [66]. Other treatment options such as mAb 35-1f4, which neutralises PV1 by virion cross-linking [67], and the capsid-binding pyridazinamine analogue R75761 [68] have been developed. In 2010, Thys et al. isolated and characterised VHHs specific to PV1 and assessed their antiviral activity in vitro.

By repeated infection of a dromedary with PV1 Sabin strain and isolation of its VHH repertoire, an immune library was created. Fifteen $\mathrm{VHH}$ clones that positively reacted with PV1 Sabin strain in an ELISA were further analysed. In a standard neutralisation assay their abilities to block infectivity of PV1 (vaccine and wild-type strain), PV2 and PV3 were determined. Neutralising activity against PV2 and PV3 was non-existent, whereas both the vaccine and wild-type strain of PV1 were neutralised by five VHHs (PVSP6A, PVSS8A, PVSP19B, PVSS21E, and PVSP29F). HeLa cell cultures were incubated with dilutions of these VHHs and cell viability was monitored, in order to reveal possible cytotoxic effects. Cytotoxicity was, however, not demonstrated for any of the tested VHHs.

Antiviral activities of the neutralising VHHs at different concentrations were assessed by examination of their cytopathic reduction effect in HeLa cells infected with PV1, and were compared with that of mAb 35-1f4 and R75761. Full protection from a PV1-induced cytopathic effect was provided by PVSP6A and PVSP29F at a concentration below that of R75761. Moreover, these VHHs exhibited protective activities comparable to mAb 35-1f4. Among the VHHs tested, the lowest half maximal effective concentration $\left(\mathrm{EC}_{50}\right.$ ) values were obtained for PVSP6A and PVSP29F.

In order to further define antiviral activities, abilities of the VHHs to reduce infectious virus yields were investigated in PV1-infected HeLa cells. Certain concentrations of PVSP6A and PVSP29F completely abolished virus replication. In 
contrast, cells treated with equal concentrations of mAb 35-1f4 showed residual virus titres.

For the generation of PV1 neutralisation escape mutants, mixtures of PV1 and VHH were created. Isolation of resistant viruses failed for PVSP6A and PVSP29F due to complete inhibition of plaque formation. The other three VHHs were unable to neutralise about 100 plaque forming units (PFU), which was consistent with the number of PFU expected for a neutralising mAb [69].

Thys et al. (2010) aimed to isolate a VHH that could inhibit cellular attachment and cell entry by binding within the canyon region. Recent studies have demonstrated that all of the PV1-neutralising VHHs recognise epitopes in the canyon region that overlap with the binding site for CD155 and thereby block ligand-receptor interactions $[70,71]$. Furthermore, PV1 neutralisation escape mutants have been found to bear amino acid substitutions in capsid VPs closely located to the VHH binding sites [72]. PVSP6A and PVSP29F were the most potent inhibitors of PV1, among the VHHs tested. Moreover, their efficacies were superior to R75761 and mAb 35-1f4 at equivalent concentrations. These VHHs could therefore be used to develop advanced antiviral drugs that prevent or treat PV1-induced poliomyelitis. Additionally, Thys et al. (2010) plan to adapt their immunisation protocols to generate a $\mathrm{VHH}$ with protective activity against all of the three PV types, with the aim to contribute to worldwide eradication of poliomyelitis.

\section{Conclusions}

VHH antibody fragments compare to conventional mAbs in their selectivity, antigen specificity and binding affinity, but excel in terms of their small size, robustness and increased solubility, which allow for construction of potent multivalent molecules with high avidity, and thus are advantageous for the development of therapeutic drugs. VHHs were found to exceed the in vivo efficacies of certain marketed drugs, especially when assembled into multimeric formats or linked to enzymes. Microbial production of VHHs is furthermore a feasible alternative to the generation of mAbs by mammalian 
402 cell culture [73] and the chemical synthesis of immunological agents. Albeit the great 403 pharmaceutical potential, no $\mathrm{VHH}$-based agents have been approved for therapy thus 404 far. However, some VHHs, such as ALX-0171, are already in advanced clinical phases. Besides their application in prevention and treatment of viral and bacterial 406 infections, VHHs have been used to combat pathologies such as arthritis [74], cancer 407 [75] and thrombosis [76], to trace antigens and biomarkers for in vivo diagnostic 408 imaging $[77,78]$, and to protect against lethal scorpion and snake envenoming $409 \quad[79,80]$.

410 Based on their versatile applicability in the biomedical field, VHHs are predicted 411 as major contributors to the solving of public health problems of the future.

$414 \quad$ Funding

$415 \quad$ None to declare.

\section{Declaration of interest}

$419 \quad$ None to declare.

\section{Acknowledgements}

$423 \quad$ None.

\section{References}

[1] Padlan EA. Anatomy of the antibody molecule. Mol. Immunol. 1994;31:169217. 
430 [2] Vidarsson G, Dekkers G, Rispens T. IgG subclasses and allotypes: from structure to effector functions. Front. Immunol. 2014;5:520.

[3] Hamers-Casterman C, Atarhouch T, Muyldermans S, et al. Naturally occuring antibodies devoid of light chains. Nature. 1993;363:446-448.

[4] Muyldermans S, Atarhouch T, Saldanha J, et al. Sequence and structure of VH domain from naturally occurring camel heavy chain immunoglobulins lacking light chains. Protein Eng. 1994;7:1129-1135.

[5] Harmsen MM, Ruuls RC, Nijman IJ, et al. Llama heavy-chain V regions consist of at least four distinct subfamilies revealing novel sequence features. Mol. Immunol. 2000;37:579-590.

[6] Vu KB, Arbabi Ghahroudi M, Wyns L, et al. Comparison of Llama VH Sequences from Conventional and Heavy Chain Antibodies. Mol. Immunol. 1997;34:11211131.

[7] Sheriff S, Constantine KL. Redefining the minimal antigen-binding fragment. Nat. Struct. Biol. 1996;3:733-736.

[8] Zhang J, Tanha J, Hirama T, et al. Pentamerization of single-domain antibodies from phage libraries: a novel strategy for the rapid generation of high-avidity antibody reagents. J. Mol. Biol. 2004;335:49-56.

[9] Chothia C, Gelfand I, Kister A. Structural determinants of immunoglobulin variable domain. J Mol Biol. 1998;278:457-479.

[10] Desmyter A, Transue TR, Ghahroudi MA, et al. Crystal structure of a camel single-domain $\mathrm{VH}$ antibody fragment in complex with lysozyme. Nat. Struct. Biol. 1996;3:803-811.

[11] Desmyter A, Decanniere K, Muyldermans S, et al. Antigen Specificity and High Affinity Binding Provided by One Single Loop of a Camel Single-domain Antibody. J. Biol. Chem. 2001;276:26285-26290.

[12] Arbabi Ghahroudi M, Desmyter A, Wyns L, et al. Selection and identification of single domain antibody fragments from camel heavy-chain antibodies. FEBS Lett. $1997 ; 414: 521-526$. 
[13] Harmsen MM, De Haard HJ. Properties, production, and applications of camelid single-domain antibody fragments. Appl. Microbiol. Biotechnol. 2007;77:13-22.

[14] Qi H, Lu H, Qiu H-J, et al. Phagemid Vectors for Phage Display: Properties, Characteristics and Construction. J Mol Biol. 2012;417:129-143.

[15] Rondof S, Koch J, Breitling F, et al. A helper phage to improve single-chain antibody presentation in phage display. Nat Biotechnol. 2001;19:75-78.

[16] Frenken LGJ, Van Der Linden RHJ, Hermans PWJJ, et al. Isolation of antigen specific Llama VHH antibody fragments and their high level secretion by Saccharomyces cerevisiae. J. Biotechnol. 2000;78:11-21.

[17] Rahbarizadeh F, Rasaee MJ, Forouzandeh M, et al. Over expression of antiMUC1 single-domain antibody fragments in the yeast Pichia pastoris. Mol. Immunol. 2006;43:426-435.

[18] Touger-Decker R, Van Loveren C. Sugars and dental caries. Am J Clin Nutr. $2003 ; 78: 881 S-892 S$

[19] Forssten SD, Björklund M, Ouwehand AC. Streptococcus mutans, caries and simulation models. Nutrients. 2010;2:290-298.

[20] Krüger C, Hultberg A, Marcotte $\mathrm{H}$, et al. Therapeutic effect of llama derived VHH fragments against Streptococcus mutans on the development of dental caries. Appl Microbiol Biotechnol. 2006;72:732-737.

[21] Russell MW, Lehner T. Characterisation of antigens extracted from cells and culture fluids of Streptococcus mutans serotype c. Arch Oral Biol. 1978;23:715.

[22] Szynol A, De Soet J], Sieben-Van Tuyl E, et al. Bactericidal Effects of a Fusion Protein of Llama Heavy-Chain Antibodies Coupled to Glucose Oxidase on Oral Bacteria. Antimicrob. Agents Chemother. 2004;48:3390-3395.

[23] Tenovuo J, Pruitt KM. Relationship of the human salivary peroxidase system to oral health. J Oral Pathol. 1984;13:573-584.

[24] Pålsson-McDermott EM, O'Neill LAJ. Signal transduction by the lipopolysaccharide receptor, Toll-like receptor-4. Immunology. 2004;113:153- 
162.

[25] Sweet MJ, Hume DA. Endotoxin signal transduction in macrophages. J Leukoc Biol. 1996;60:8-26.

[26] Cohen J. The immunopathogenesis of sepsis. Nature. 2002;420:885-891.

[27] Rosenstein NE, Perkins BA, Stephens DS, et al. Meningococcal Disease. N. Engl. J. Med. $2001 ; 344: 1378-1388$.

[28] Scholten RJ, Kuipers B, Valkenburg HA, et al. Lipo-oligosaccharide immunotyping of Neisseria meningitidis by a whole-cell ELISA with monoclonal antibodies. J. Med. Microbiol. 1994;41:236-243.

[29] Tsai CM, Boykins R, Frasch CE. Heterogeneity and variation among Neisseria meningitidis lipopolysaccharides. J. Bacteriol. 1983;155:498-504.

[30] Pollack M, Raubitschek AA, Larrick JW. Human monoclonal antibodies that recognize conserved epitopes in the core-lipid A region of lipopolysaccharides. J. Clin. Invest. $1987 ; 79: 1421-1430$.

[31] Di Padova FE, Brade H, Barclay GR, et al. A broadly cross-protective monoclonal antibody binding to Escherichia coli and Salmonella lipopolysaccharides. Infect. Immun. 1993;61:3863-3872.

[32] El Khattabi M, Adams H, Heezius E, et al. Llama Single-Chain Antibody That Blocks Lipopolysaccharide Binding and Signaling: Prospects for Therapeutic Applications. Clin. Vaccine Immunol. 2006;13:1079-1086.

[33] Berberov EM, Zhou Y, Francis DH, et al. Relative importance of heat-labile enterotoxin in the causation of severe diarrheal disease in the gnotobiotic piglet model by a strain of enterotoxigenic Escherichia coli that produces multiple enterotoxins. Infect. Immun. 2004;72:3914-3924.

[34] Nataro JP, Kaper JB. Diarrheagenic Escherichia coli. Clin. Microbiol. Rev. $1998 ; 11: 142-201$.

[35] Nair B, Takeda Y. The heat-stable enterotoxins. Microb. Pathog. 1998;24:123131.

[36] Van den Broeck W, Cox E, Oudega B, et al. The F4 fimbrial antigen of 
Escherichia coli and its receptors. Vet. Microbiol. 2000;71:223-244.

[37] Tzipori S, Chandler D, Smith M, et al. Factors contributing to postweaning diarrhoea in a large intensive piggery. Aust. Vet. J. 1980;56:274-278.

[38] Osek J. Detection of the enteroaggregative Escherichia coli heat-stable enterotoxin 1 (EAST1) gene and its relationship with fimbrial and enterotoxin markers in E. coli isolates from pigs with diarrhoea. Vet. Microbiol. 2003;91:6572.

[39] Harmsen MM, Van Solt CB, Hoogendoorn A, et al. Escherichia coli F4 fimbriae specific llama single-domain antibody fragments effectively inhibit bacterial adhesion in vitro but poorly protect against diarrhoea. Vet. Microbiol. $2005 ; 111: 89-98$.

[40] Macasaet FF, Kidd PA, Bolano CR, et al. The etiology of acute respiratory infections. 3. The role of viruses and bacteria. J. Pediatr. 1968;72:829-839.

[41] Backman K, Piippo-Savolainen E, Ollikainen $\mathrm{H}$, et al. Adults face increased asthma risk after infant RSV bronchiolitis and reduced respiratory health-related quality of life after RSV pneumonia. Acta Paediatr. 2014;103:850-855.

[42] Sikkel MB, Quint JK, Mallia P, et al. Respiratory Syncytial Virus Persistence in Chronic Obstructive Pulmonary Disease. Pediatr. Infect. Dis. J. 2008;27:S63S70.

[43] Zhang L, Peeples ME, Boucher RC, et al. Respiratory syncytial virus infection of human airway epithelial cells is polarized, specific to ciliated cells, and without obvious cytopathology. J. Virol. 2002;76:5654-5666.

[44] Sterner G, Wolontis S, Bloth B, et al. Respiratory syncytial virus. An outbreak of acute respiratory illnesses in a home for infants. Acta Paediatr. Scand. $1966 ; 55: 273-279$.

[45] Huang YT, Wertz GW. The genome of respiratory syncytial virus is a negativestranded RNA that codes for at least seven mRNA species. J. Virol. $1982 ; 43: 150-157$.

[46] Levine S, Klaiber-Franco R, Paradiso PR. Demonstration that Glycoprotein G Is 
the Attachment Protein of Respiratory Syncytial Virus. J. Gen. Virol. $1987 ; 68: 2521-2524$.

[47] Walsh EE, Hruska J. Monoclonal antibodies to respiratory syncytial virus proteins: identification of the fusion protein. J. Virol. 1983;47:171-177.

[48] Techaarpornkul S, Barretto N, Peeples ME. Functional analysis of recombinant respiratory syncytial virus deletion mutants lacking the small hydrophobic and/or attachment glycoprotein gene. J. Virol. 2001;75:6825-6834.

[49] Peret TC, Golub JA, Anderson LJ, et al. Circulation patterns of genetically distinct group A and B strains of human respiratory syncytial virus in a community. J. Gen. Virol. 1998;79:2221-2229.

[50] Johnson PR, Olmsted RA, Prince GA, et al. Antigenic relatedness between glycoproteins of human respiratory syncytial virus subgroups A and B: evaluation of the contributions of $\mathrm{F}$ and $\mathrm{G}$ glycoproteins to immunity. J. Virol. $1987 ; 61: 3163-3166$.

[51] Impact-RSV Study Group. Palivizumab, a humanized respiratory syncytial virus monoclonal antibody, reduces hospitalization from respiratory syncytial virus infection in high-risk infants. Pediatrics. 1998;102:531-537.

[52] Detalle L, Stohr T, Palomon C, et al. Generation and Characterization of ALX0171, a Potent Novel Therapeutic Nanobody for the Treatment of Respiratory Syncytial Virus Infection. Antimicrob. Agents Chemother. 2016;60:6-13.

[53] Ablynx. Results from the first-in-infant Phase I/IIa study with the anti-RSV Nanobody, ALX-0171 [Internet]. ALX-0171. 2016 [cited 2016 Nov 23]. Available from: http://www.ablynx.com/uploads/data/files/ablynx_alx-0171_first-ininfant study results_webcast presentation.pdf.

[54] Landsteiner K, Popper E. Übertragung der Poliomyelitis acuta auf Affen. Zeitschr Immunitätsforsch. 1909;2:377-390.

[55] Bodian D, Morgan IM, Howe HA. Differentiation of types of poliomyelitis viruses; the grouping of 14 strains into three basic immunological types. Am. J. Hyg. $1949 ; 49: 234-245$. 
[56] Kitamura N, Semler BL, Rothberg PG, et al. Primary structure, gene organization and polypeptide expression of poliovirus RNA. Nature. $1981 ; 291: 547-553$.

[57] Maizel J V, Summers DF. Evidence for differences in size and composition of the poliovirus-specific polypeptides in infected HeLa cells. Virology. 1968;36:48-54.

[58] Hogle JM, Chow M, Filman DJ. Three-dimensional structure of poliovirus at $2.9 \mathrm{~A}$ resolution. Science (80-. ). 1985;229:1358-1365.

[59] Mendelsohn CL, Wimmer E, Racaniello VR. Cellular receptor for poliovirus: molecular cloning, nucleotide sequence, and expression of a new member of the immunoglobulin superfamily. Cell. 1989;56:855-865.

[60] Colston E, Racaniello VR. Soluble receptor-resistant poliovirus mutants identify surface and internal capsid residues that control interaction with the cell receptor. EMBO J. 1994;13:5855-5862.

[61] Rossmann MG. Viral cell recognition and entry. Protein Sci. 1994;3:1712-1725.

[62] Fricks CE, Hogle JM. Cell-induced conformational change in poliovirus: externalization of the amino terminus of VP1 is responsible for liposome binding. J. Virol. 1990;64:1934-1945.

[63] Bodian D. Emerging concept of poliomyelitis infection. Science (80-. ). $1955 ; 122: 105-108$.

[64] Salk JE. Studies in Human Subjects on Active Immunization against Poliomyelitis. I. A Preliminary Report of Experiments in Progress. J. Am. Med. Assoc. $1953 ; 151: 1081-1098$.

[65] Sabin AB. Present status of attenuated live-virus poliomyelitis vaccine. J. Am. Med. Assoc. 1956;162:1589-1596.

[66] Nathanson N, Kew OM. From emergence to eradication: the epidemiology of poliomyelitis deconstructed. Am. J. Epidemiol. 2010;172:1213-1229.

[67] Thomas AA, Brioen P, Boeyé A. A monoclonal antibody that neutralizes poliovirus by cross-linking virions. J. Virol. 1985;54:7-13.

[68] Thys B, De Palma AM, Neyts J, et al. R75761, a lead compound for the 
development of antiviral drugs in late stage poliomyelitis eradication strategies and beyond. Antiviral Res. 2008;78:278-281.

[69] Thys B, Schotte L, Muyldermans S, et al. In vitro antiviral activity of single domain antibody fragments against poliovirus. Antiviral Res. 2010;87:257-264.

[70] Strauss M, Schotte L, Thys B, et al. Five of Five VHHs Neutralizing Poliovirus Bind the Receptor-Binding Site. J. Virol. 2016;90:3496-3505.

[71] Schotte L, Strauss M, Thys B, et al. Mechanism of action and capsid-stabilizing properties of VHHs with an in vitro antipolioviral activity. J. Virol. $2014 ; 88: 4403-4413$.

[72] Schotte L, Thys B, Strauss M, et al. Characterization of Poliovirus Neutralization Escape Mutants of Single-Domain Antibody Fragments (VHHs). Antimicrob. Agents Chemother. 2015;59:4695-4706.

[73] Seifert DB, Phillips JA. The production of monoclonal antibody in growtharrested hybridomas cultivated in suspension and immobilized modes. Biotechnol. Prog. 1999;15:655-666.

[74] Coppieters K, Dreier T, Silence K, et al. Formatted anti-tumor necrosis factor a VHH proteins derived from camelids show superior potency and targeting to inflamed joints in a murine model of collagen-induced arthritis. Arthritis Rheum. $2006 ; 54: 1856-1866$.

[75] Roovers RC, Laeremans T, Huang L, et al. Efficient inhibition of EGFR signaling and of tumour growth by antagonistic anti-EFGR Nanobodies. Cancer Immunol. Immunother. 2007;56:303-317.

[76] Ulrichts H, Silence K, Schoolmeester A, et al. Antithrombotic drug candidate ALX-0081 shows superior preclinical efficacy and safety compared with currently marketed antiplatelet drugs. Blood. 2011;118:757-765.

[77] Rothbauer U, Zolghadr K, Tillib S, et al. Targeting and tracing antigens in live cells with fluorescent nanobodies. Nat. Methods. 2006;3:887-890.

[78] Huang L, Gainkam LOT, Caveliers V, et al. SPECT imaging with 99mTc-labeled EGFR-specific nanobody for in vivo monitoring of EGFR expression. Mol. Imaging 
1

2

3

4

5

6

7

8

9

10

11

12
Biol. 2008; 10:167-175.

[79] Hmila I, Saerens D, Ben Abderrazek R, et al. A bispecific nanobody to provide full protection against lethal scorpion envenoming. FASEB J. 2010;24:34793489.

[80] Chavanayarn C, Thanongsaksrikul J, Thueng-in K, et al. Humanized-Single Domain Antibodies (VH/VHH) that Bound Specifically to Naja kaouthia Phospholipase A2 and Neutralized the Enzymatic Activity. Toxins (Basel). $2012 ; 4: 554-567$. 